\title{
Spectrum Assignment Algorithm for Cognitive Machine-to-Machine Networks
}

\author{
Soheil Rostami, ${ }^{1}$ Sajad Alabadi, ${ }^{1}$ Soheir Noori, ${ }^{2}$ Hayder Ahmed Shihab, ${ }^{3}$ \\ Kamran Arshad, ${ }^{4}$ and Predrag Rapajic ${ }^{1}$
}

${ }^{1}$ Department of Engineering Science, University of Greenwich, London, UK

${ }^{2}$ Department of Computer Science, University of Karbala, Karbala, Iraq

${ }^{3}$ School of Engineering and Informatics, University of Sussex, Brighton, UK

${ }^{4}$ Department of Electrical Engineering, Ajman University of Science \& Technology, Ajman, UAE

Correspondence should be addressed to Soheil Rostami; s.rostami@gre.ac.uk

Received 18 March 2016; Revised 15 June 2016; Accepted 10 July 2016

Academic Editor: Fernando Casadevall

Copyright (C) 2016 Soheil Rostami et al. This is an open access article distributed under the Creative Commons Attribution License, which permits unrestricted use, distribution, and reproduction in any medium, provided the original work is properly cited.

A novel aggregation-based spectrum assignment algorithm for Cognitive Machine-To-Machine (CM2M) networks is proposed. The introduced algorithm takes practical constraints including interference to the Licensed Users (LUs), co-channel interference (CCI) among CM2M devices, and Maximum Aggregation Span (MAS) into consideration. Simulation results show clearly that the proposed algorithm outperforms State-Of-The-Art (SOTA) algorithms in terms of spectrum utilisation and network capacity. Furthermore, the convergence analysis of the proposed algorithm verifies its high convergence rate.

\section{Introduction}

Today, there are around 4 billion M2M devices in the world, while in 2022, the number is expected to reach 50 billion [1]. According to Cisco systems, currently a single M2M device can generate as much traffic as 3 basic-feature phones; in addition, emerging applications and services of M2M networks are expected to increase average traffic per device from $70 \mathrm{MB}$ per month in 2014 to $366 \mathrm{MB}$ per month in 2018 [2]. Because of the growth rate of the number of devices and high demand of data traffic, future M2M networks will face many challenges, especially with the so-called spectrum scarcity problem.

Cognitive Radio (CR) is introduced as a promising solution to tackle spectrum scarcity problem in M2M networks. CR has become one of the most intensively studied paradigms in wireless communications. In CR, unlicensed users exploit CR technology to opportunistically access licensed spectrum as long as interference to LUs is kept at an acceptable level [3]. A number of M2M applications (such as smart grid, healthcare, and car parking) can benefit from the combination of $\mathrm{CR}$ and M2M communications [1]. CM2M networks can improve spectrum utilisation and energy efficiency in M2M networks [4]. The CM2M device can interact with the radio environment by either performing spectrum sensing or accessing spectrum databases or both of them to detect spectrum opportunities [4]. After sensing, CM2M device utilises the discovered unused spectrum according to the device requirements.

Furthermore, TV bands (VHF/UHF), which have highly favourable propagation characteristics, are traditionally reserved to broadcasters. But after the transition from the analogue broadcast television system to the digital one, a huge number of TV channels (also known as TV White Spaces (TVWS)) are freed up and unused. In September 2010, the Federal Communications Commission (FCC) released significant rule to enable unlicensed broadband wireless devices to use TVWS. Unfortunately, due to spectrum fragmentation and as a result of an inefficient command and control spectrum management approach, a continuous wide segment of TVWS is rare in many countries including the United Kingdom. 


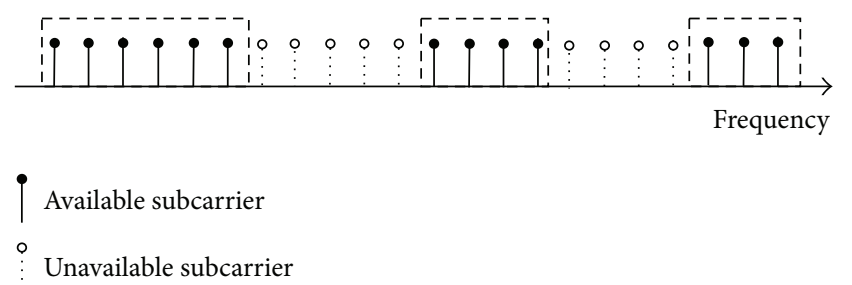

FIGURE 1: Subcarrier distribution over spectrum [7].

As CM2M network can sense and be aware of its radio environment, the aggregation of narrow spectrum opportunities becomes possible. Spectrum aggregation provides wider bandwidth and higher throughput for the CM2M devices. CM2M devices can access discontinuous portions of the TVWS simultaneously by means of Discontinuous Orthogonal Frequency Division Multiplexing (DOFDM) [5, $6]$.

DOFDM is a multicarrier modulation technique and is a variant of OFDM used to aggregate discontinuous segments of spectrum. The main difference between OFDM and DOFDM is ON/OFF subcarrier information block [7]. A multiple segments of spectrum can be occupied by other CM2M devices or LUs. As a result, these subcarriers are offlimits to the CM2M devices [6]. Thus, to avoid interfering with these other transmissions, the subcarrier within their vicinity is turned off and unusable for CM2M devices, as shown in Figure 1. Moreover, available (usable) subcarriers are located in the unoccupied segments of spectrum, which are determined by spectrum broker.

Spectrum aggregation is one of the most important LTEadvanced technologies from physical layer perspective and standardised in LTE Release 10 [8]. However, in spite of standardisation of spectrum aggregation, little effort has been made to optimise spectrum aggregation by exploiting CR technology in M2M networks. There is limited literature available on spectrum assignment among CM2M devices having spectrum aggregation capabilities.

In [9], an Aggregation-Aware Spectrum Assignment Algorithm (AASAA) is proposed to aggregate discrete spectrum fragments in a greedy manner. The algorithm in [9] utilises the first available aggregation range from the low frequency side and assumes that all users have the same bandwidth requirement.

Huang et al. [10] proposed a prediction based spectrum aggregation scheme to increase the capacity and decrease the reallocation overhead. The proposed scheme is referred to as Maximum Satisfaction Algorithm (MSA) for spectrum assignment. The main idea is to assign spectrum for the user with larger bandwidth requirement first, leaving better spectrum bands for remaining users, while taking into consideration different bandwidth requirements of users and channel state statistics. However, MSA does not enhance spectrum utilisation by reusing spectrum within unlicensed network; that is, CCI is neglected in MSA.

Recently, genetic algorithm (GA) is used for spectrum allocation [11]. Ye et al. [11] introduced a GA based spectrum assignment in CR networks; but spectrum aggregation capability of users is not considered.

For CM2M networks, existing spectrum assignment and aggregation solutions are not applicable directly as practical issues such as Maximum Aggregation Span (MAS) must be taken into account. Furthermore, in aggregation-based spectrum assignment a major challenge is to manage CCI among CM2M devices which is not taken into account in the existing literature. The major contributions of this study are twofold.

(1) To prevent multiple CM2M devices from colliding in the overlapping portions of the spectrum, a centralised approach is applied. Furthermore, an integer optimisation problem to maximise cell throughput is formulated, considering CCI and MAS in an aggregation-aware CM2M network.

(2) As the spectrum assignment problem is inherently seen as an NP-hard optimisation problem, evolutionary approaches can be applied to solve this challenging problem. In this article, GA is used to solve the aggregation-aware spectrum assignment because of its simplicity, robustness, and fast convergence of the algorithm [12].

This article is organised as follows. In Section 2, the spectrum assignment and aggregation models are presented. The proposed algorithm is explained in Section 3. Simulation results are discussed in Section 4, followed by conclusions in Section 5 .

\section{System Model}

2.1. Spectrum Assignment Model. We assume a CM2M network consisting of $N$ CM2M devices defined as $\Phi=$ $\left\{\phi_{1}, \phi_{2}, \ldots, \phi_{N}\right\}$ competing for $M$ nonoverlapping orthogonal channels $\Gamma=\left\{\gamma_{1}, \gamma_{2}, \ldots, \gamma_{M}\right\}$ in uplink. All spectrum assignment and access procedures are controlled by a central entity called spectrum broker. We assume that distributed sensing mechanism and measurement conducted by each device is forwarded to the spectrum broker [13]. A spectrum occupancy map that is constructed at the spectrum broker and CCI among CM2M devices is determined. Furthermore, the spectrum broker can lease single or multiple channels for $\phi_{n} \in \Phi$ in a limited geographical region for a certain amount of time. Finally, a base station can transmit data to $\phi_{n}$ in the assigned channels. Figure 2 depicts system model used in this article.

We define the channel availability matrix $\mathbf{L}=\left\{l_{n, m} \mid l_{n, m} \in\right.$ $\{0,1\}\}_{N \times M}$ as an $N \times M$ binary matrix representing channel availability, where $l_{n, m}=1$ if and only if $\gamma_{m}$ is available to $\phi_{n}$ and $l_{n, m}=0$ otherwise. Each $\phi_{n}$ is associated with a set of available channels at its location defined as $\bar{\Gamma}_{n} \subset \Gamma$; that is, $\bar{\Gamma}_{n}=\left\{\gamma_{m} \mid l_{n, m} \neq 0\right\}$. Due to the different interference range of each LU (which depends on LU's transmit power and the physical distance) at the location of each CM2M device, $\bar{\Gamma}_{n}$ of different CM2M devices may be different [14]. According to the sharing agreement, any $\gamma_{m} \in \Gamma$ can be reused by a group of CM2M devices in the vicinity defined by $\bar{\Phi}_{m}$ such that $\bar{\Phi}_{m} \subset$ 


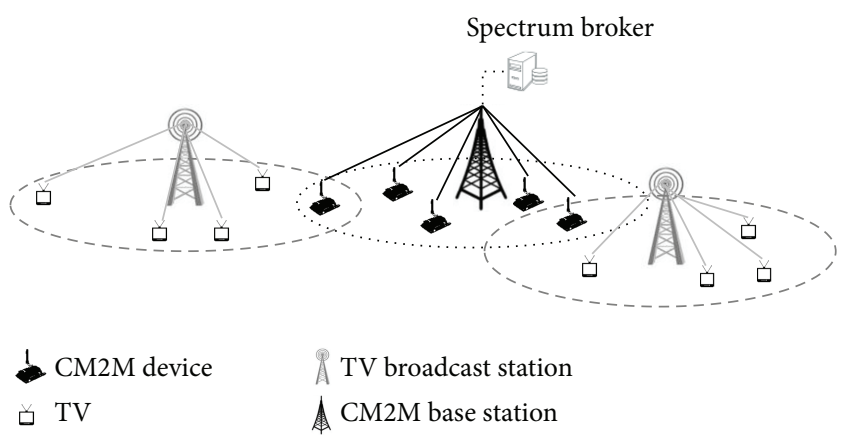

FIGURE 2: Architecture diagram of CM2M network operating in TVWS.

$\Phi$, if CM2M devices are located outside the interference range of LUs; that is, $\bar{\Phi}_{m}=\left\{\phi_{n} \mid l_{n, m} \neq 0\right\}$.

The interference constraint matrix $\mathbf{C}=\left\{c_{n, k, m} \mid c_{n, k, m} \in\right.$ $\{0,1\}\}_{N \times N \times M}$ is an $N \times N \times M$ binary matrix representing the interference constraint among CM2M devices, where $c_{n, k, m}=$ 1 if $\phi_{n}$ and $\phi_{k}$ would interfere with each other on $\gamma_{m}$, and $c_{n, k, m}=0$ otherwise. It should be noted that for $n=k, c_{n, n, m}=$ $1-l_{n, m}$. Value of $c_{n, k, m}$ depends on the distance between $\phi_{n}$ and $\phi_{k}$. Interference constraint also depends on $\gamma_{m}$ as power and transmission rules vary greatly in different frequency bands. The bandwidth requirements of all CM2M devices are diverse because of different quality of service requirements for each device. We define $\mathscr{R}=\left\{r_{n}\right\}_{1 \times N}$ as device requested bandwidth vector, where $r_{n}$ represents bandwidth demand of $\phi_{n}$.

In a dynamic environment, channels availability and interference constraint matrix both vary continually; in this study, we assume that spectrum availability is static or varies slowly in each scheduling time slot; that is, all matrices remain constant during the scheduling period. In our proposed solution, a subset of CM2M devices is scheduled during each time slot and the available spectrum is allocated among them without causing interference to LUs.

2.2. Spectrum Aggregation Model. In the traditional spectrum assignment, each channel is composed of a continuous spectrum fragment; thus it is not feasible for users to utilise small spectrum fragments which are smaller than the users bandwidth demand. For instance, assume a CM2M network where every machine requires $4 \mathrm{MHz}$ channel bandwidth, and the available spectrum consists of two spectrum fragments of $4 \mathrm{MHz}$ and four spectrum fragments of $2 \mathrm{MHz}$ (Figure 3). For continuous spectrum allocation, the $2 \mathrm{MHz}$ spectrum fragments cannot be utilised by any machine. Therefore, a continuous spectrum assignment mode can only support two devices for communication $(2 \times 4 \mathrm{MHz})$. However, spectrum aggregation-enabled device can exploit fragmented segments of the spectrum by using specialised air interface techniques, such as DOFDM. In Figure 3, if a number of small spectrum fragments are aggregated into a wider channel, then $16 \mathrm{MHz}$ of unused spectrum is available to support four CM2M devices $(4 \times 4 \mathrm{MHz})$.

Due to the limited aggregation capabilities of the RF front-end, only channels that reside within a range of MAS can be aggregated. With this constraint, some spectrum fragments may not be aggregated because their span is larger than MAS. Our proposed algorithm takes MAS into consideration. For the sake of simplicity, we make following assumptions.

(1) All CM2M devices have the same aggregation capability (i.e., MAS for all devices is the same).

(2) Guard band between adjacent channels is neglected.

(3) Bandwidth requirement of each device and bandwidth of each channel are an integer multiple of subchannel bandwidth $\Delta$, which is the smallest unit of bandwidth (in fact, the smaller fragments would demand excessive filtering to limit adjacent channel interference); that is,

$$
\begin{aligned}
r_{n} & =\omega_{n} \cdot \Delta, \quad \omega_{n} \in \mathbb{N}, 1 \leq n \leq N, \\
\mathscr{B} \mathscr{W}_{m} & =\kappa_{m} \cdot \Delta, \quad \kappa_{m} \in \mathbb{N}, 1 \leq m \leq M,
\end{aligned}
$$

where $\mathbb{N}$ is the set of natural numbers, $\omega_{n}$ is the number of requested subchannels by $\phi_{n}, \kappa_{m}$ is the number of subchannels in $\gamma_{m}$, and $\mathscr{B} \mathscr{W}_{m}$ is the bandwidth of $\gamma_{m}$.

The total available spectrum (i.e., $M$ channels) is subdivided into multiple number of subchannels. If the available spectrum band consists of $\mathscr{C}$ subchannels (i.e., total available bandwidth is $\mathscr{C} \cdot \Delta$ ), then

$$
\begin{aligned}
& \gamma_{m}=\bigcup_{i=1}^{\kappa_{m}} \tilde{\gamma}_{i, m}, \\
& \kappa_{m}=\frac{\mathscr{B} \mathscr{W}_{m}}{\Delta}, \\
& \text { where } 1 \leq m \leq M, \\
& \mathscr{C}=\sum_{m=1}^{M} \kappa_{m},
\end{aligned}
$$

where $\gamma_{m}$ has $\kappa_{m}$ subchannels and $\tilde{\gamma}_{i, m}$ represents the $i$ th subchannel of $\gamma_{m}$. Each $\tilde{\gamma}_{i, m}$ can be represented in an interval defined as $\left[\mathscr{F}_{i, m}^{L}, \mathscr{F}_{i, m}^{H}\right]$, where $\mathscr{F}_{i, m}^{L}$ and $\mathscr{F}_{i, m}^{H}$ are the lowest and highest frequency of $\widetilde{\gamma}_{i, m}$ :

$$
\mathscr{F}_{i, m}^{H}-\mathscr{F}_{i, m}^{L}=\Delta, \quad \text { for } 1 \leq i \leq \kappa_{m}, \quad 1 \leq m \leq M .
$$

Based on this new subchannel indexing, matrices $\mathbf{L}$ and $\mathbf{C}$ can be rewritten as

$$
\begin{aligned}
& \mathbf{L}^{*}=\left\{l_{n, \mathrm{c}}^{*} \mid l_{n, \mathrm{c}}^{*}=l_{n, m}\right\}_{N \times \mathscr{C}}, \\
& \mathbf{C}^{*}=\left\{c_{n, k, \mathrm{c}}^{*} \mid c_{n, k, \mathrm{c}}^{*}=c_{n, k, m}\right\}_{N \times N \times \mathscr{C}}
\end{aligned}
$$

if

$$
\begin{gathered}
1 \leq \mathrm{c} \leq \kappa_{1} \quad \text { for } m=1, \\
\sum_{j=1}^{m-1} \kappa_{j}<\mathrm{c} \leq \sum_{j=1}^{m} \kappa_{j} \quad \text { for } 1<m \leq M,
\end{gathered}
$$




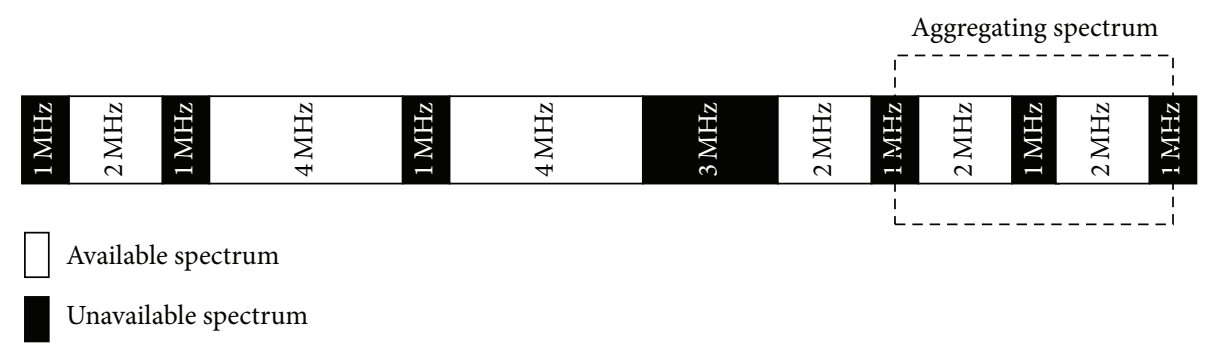

FIGURE 3: Aggregation of disjoint spectrum fragments.

where c represents index of each subchannel within the available spectrum.

The subchannel assignment matrix $\mathbf{A}=\left\{a_{n, \mathrm{c}} \mid a_{n, \mathrm{c}} \in\right.$ $\{0,1\}\}_{N \times \mathscr{C}}$ is an $N \times \mathscr{C}$ binary matrix representing subchannels assigned to CM2M devices for aggregation such that $a_{n, \mathrm{c}}=1$ if and only if subchannel $c$ is available to $\phi_{n}$ and 0 otherwise. We define the reward vector $\mathscr{B}=\left\{b_{n}=\Delta \cdot \sum_{c}^{\mathscr{C}} a_{n, \mathrm{c}}\right\}_{N \times 1}$ to represent total bandwidth that is allocated to each CM2M device during scheduling time period for a given subchannel assignment.

\section{Problem Formulation}

3.1. Optimisation Problem. One of the key objectives of the deployment of CM2M network is to enhance the spectrum utilisation. To consider this crucial goal, we define network utilisation to maximise the total bandwidth that is assigned to CM2M devices and referred to as Maximising Sum of Reward (MSR):

$$
\operatorname{MSR}=\sum_{n=1}^{N} b_{n} .
$$

To maximise MSR the spectrum aggregation problem can be defined as a constrained optimisation problem as follows:

$$
\max _{a} \sum_{n=1}^{N} b_{n}
$$

$$
\begin{aligned}
\text { subject to } \quad b_{n}=\Delta \cdot \sum_{\mathrm{c}=1}^{\mathscr{C}} a_{n, \mathrm{c}} \\
= \begin{cases}0 & \text { if } \phi_{n} \text { is rejected, } \\
r_{n} & \text { if } \phi_{n} \text { is accepted. }\end{cases}
\end{aligned}
$$

for $1 \leq n \leq N$,

$$
\begin{aligned}
& \quad \mathscr{F}_{d, t}^{H}-\mathscr{F}_{e, f}^{L} \leq \mathrm{MAS}, \\
& a_{n, \mathrm{c}}=0 \\
& \quad \text { if } l_{n, \mathrm{c}}^{*}=0 \text {, for } 1 \leq n \leq N, 1 \leq \mathrm{c} \leq \mathscr{C}, \\
& a_{n, \mathrm{c}} \cdot a_{k, \mathrm{c}}=0 \\
& \text { if } c_{n, k, \mathrm{c}}^{*}=1 \text {, for } 1 \leq n, k \leq N, 1 \leq \mathrm{c} \leq \mathscr{C} .
\end{aligned}
$$

Expression (8) assures that rewarded bandwidth $b_{n}$ to each accepted $\phi_{n}$ must be equal to $\phi_{n}$ 's bandwidth demand $r_{n}$; if CM2M network cannot satisfy $\phi_{n}$ 's bandwidth request, $\phi_{n}$ is rejected and $b_{n}=0$. If $\mathscr{F}_{e, f}^{L}\left(1 \leq e \leq \kappa_{f}\right.$ and $\left.1 \leq f \leq M\right)$ is the lowest frequency of an initial aggregated subchannel and $\mathscr{F}_{d, t}^{H}\left(1 \leq d \leq \kappa_{t}\right.$ and $\left.1 \leq \mathrm{t} \leq M\right)$ is the highest frequency of a terminative subchannel, (9) guarantees that the range of allocated spectrum is equal to or less than MAS. A must satisfy the interference constraints (10) and (11); expressions (10) and (11) guarantee that there is no harmful interference to LUs and other CM2M devices, respectively.

3.2. Spectrum Aggregation Algorithm Based on Genetic Algorithm. Traditionally the spectrum assignment problem has been classified as an NP-hard problem [12]. Herein, GA is employed to solve the aggregation-based spectrum assignment problem in order to obtain faster convergence. GA is a stochastic search method that mimics the process of natural evolution. In addition, it is easy to encode solutions of spectrum assignment problem to chromosomes in GA and compare the fitness value of each solution. The specific operations of the proposed algorithm, referred to as MSR Algorithm (MSRA), can be described through the following steps.

(1) Encoding. In MSRA, a chromosome represents a possible conflict-free subchannel assignment. In order to decrease search space (by reducing redundancy in the data) and obtain faster solutions, similar approach as described in [12] is adopted in this article. We apply a mapping process between $\mathbf{A}$ and the chromosomes, based on the characteristics of $\mathbf{L}^{*}$ and $\mathbf{C}^{*}$. Only those elements of $\mathbf{A}$ are encoded whose corresponding elements in $\mathbf{L}^{*}$ take the value of 1 ; that is, $a_{n, \mathrm{c}}=0$, where $(n, c)$ satisfies $l_{n, c}^{*}=0$. As a result of this mapping, the chromosome length is equal to the number of nonzero elements of $\mathbf{L}^{*}$ and the search space is greatly reduced. Based on a given $\mathbf{L}^{*}$, length of the chromosome can be calculated as $\sum_{i=1}^{N} \sum_{j=1}^{\mathscr{C}} l_{i, j}^{*}$.

(2) Initialisation. During initialisation process, the initial population is randomly generated based on a binary coding mechanism as applied in [12]. The size of the population depends on $|\Phi|$ and $|\Gamma|$; for larger $|\Phi|$ and $|\Gamma|$, population size should be increased, where $|\cdot|$ indicates cardinality of a set. 
(3) Selection. The fitness value of each individual of the current population according to MSRA criteria defined in (6) is computed. According to the individuals fitness value, excellent individuals are selected and remain in the next generation. The chromosome with largest fitness value replaces the one with a small fitness value by the selection process.

(4) Genetic Operators. To maintain high fitness values of all chromosomes in a successive population, the crossover and mutation operators are applied. Two randomly selected chromosomes are chosen in each iteration as the parents and the crossover of the parent chromosomes is carried out at probability of crossover rate. In addition to selection and crossover operations, mutation at certain mutation rate is performed to maintain genetic diversity.

(5) Termination. The stop criteria of GA are checked in each iteration. If they can not be satisfied, step (3) and step (4) are repeated. The number of maximum iterations and the difference of fitness value are used as the criteria to determine the termination of GA.

The population of chromosomes generated after initialisation, selection, crossover, and mutation may not satisfy the given constraints defined in (8)-(11). To find feasible chromosomes that satisfy all constraints, a constraint-free process is applied that has the following steps (in order).

(1) Bandwidth Requirements. The vector $\mathscr{B}$ as given in Section 2.2 is calculated. $b_{n}$ should be equal to either $r_{n}$ or zero; otherwise all genomes related to $\phi_{n}$ are changed to zero.

(2) MAS. To satisfy the hardware limitations of the transceiver, expression (9) should be satisfied; otherwise all genomes related to $\phi_{n}$ are changed to zero.

(3) No Interference to LUs. Expression (10) guarantees that CM2M devices transmissions do not interfere LUs transmissions, ensuring that CM2M network does not harm LUs performance. If expression (10) is not satisfied, all genomes related to $\phi_{n}$ are changed to zero.

(4) CCI. Expression (11) guarantees that there is no harmful interference to other CM2M devices. If expression (11) is not satisfied, one of two conflicted devices is chosen at random, and then all genomes of the selected device are changed to zero.

To achieve higher spectrum utilisation and faster convergence, after each generation, MSRA assigns all unassigned spectra to remaining CM2M devices randomly, whenever possible. At the same time, MSRA guarantees that all the constraints defined in (8)-(11) are satisfied at all time.

\section{Simulation Results}

In this section, a set of system-level performance results are presented in order to compare and show the efficiency of MSRA over MSA [10], AASAA [9], and RCAA. The simulation results demonstrate high potential of the proposed
TABLE 1: Simulation parameters.

\begin{tabular}{lc}
\hline Parameter & Value \\
\hline$\Delta$ & $1 \mathrm{MHz}$ \\
MAS & $40 \mathrm{MHz}$ \\
$\mathscr{B} \mathscr{W}_{m}$ & $\Delta \cdot U(1,20)$ \\
$r_{n}$ & $\Delta \cdot U(1,20)$ \\
Total transmit power & $26 \mathrm{dBm}(400 \mathrm{~mW})$ \\
Scheduling time slot & $1 \mathrm{~ms}$ \\
Traffic model & Backlogged \\
Population size & 20 \\
Number of generations & 10 \\
Mutation rate & 0.01 \\
Crossover rate & 0.8 \\
\hline
\end{tabular}

method in terms of spectrum utilisation and system capacity. To assess the performance of network, independent of each device's traffic distribution model, backlogged traffic model (known as full-buffer model) is used where packet queue length of every device is much longer than what can be scheduled during each scheduling time slot.

Due to the random nature of the channel bandwidth and the devices bandwidth demand, Monte Carlo simulations are performed and each simulation scenario is repeated 100,000 times. The default parameters used in the simulations are listed in Table 1, where $U(1,20)$ represents the discrete uniform random integer numbers between 1 and 20. Each of the channels is modeled as flat Rayleigh channel with path loss model of PL $=128.1+37.6 \log _{10} R(R$ is in $\mathrm{km})$ and penetration loss of $20 \mathrm{~dB}$. The mean and standard deviation of log-normal fading are zero and $8 \mathrm{~dB}$, respectively. In our simulation model, the CM2M devices located randomly without restrictions within a rectangular area of $2 \mathrm{~km} \times 1 \mathrm{~km}$. All channels are randomly selected between $54 \mathrm{MHz}$ and $806 \mathrm{MHz}$ television frequencies (channels 2-69). Typically, the number of M2M devices is very high in each cell, but in this study, because of high computational complexity of SOTA solutions, smaller number of M2M devices is considered for comparison purposes.

To investigate the simulation results effectively, the following terms are defined and used in our analysis.

(1) Spectrum Utilisation. It is referred to as $\mathcal{U}$ which is defined as the ratio of the sum of rewarded bandwidth to the sum of all available bandwidths; that is,

$$
\mathscr{U}=\frac{\sum_{n=1}^{N} b_{n}}{\sum_{m=1}^{M} \mathscr{B} \mathscr{W}_{m}} .
$$

(2) Network Load. It is referred to as $\mathscr{L}$ which is defined as the ratio of the sum of all CM2M devices bandwidth requirements to the sum of all available bandwidths; that is,

$$
\mathscr{L}=\frac{\sum_{n=1}^{N} r_{n}}{\sum_{m=1}^{M} \mathscr{B} \mathscr{W}_{m}} .
$$




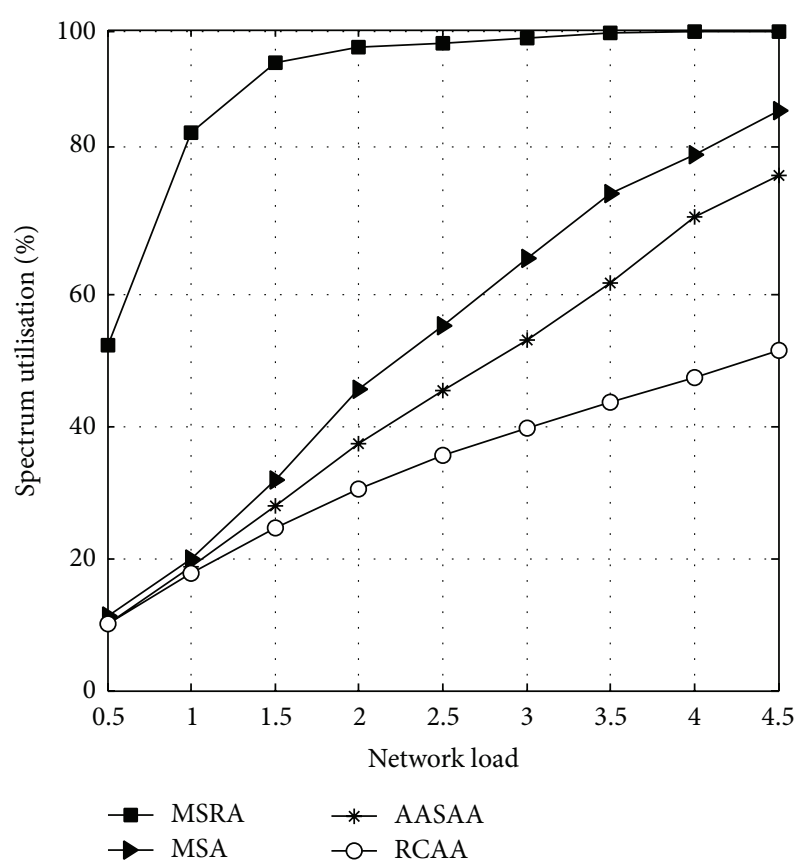

FIgURE 4: The impact of varying network load conditions on spectrum utilisation (scenario I: without CCI).

(3) Number of Rejected Devices. Rejected devices are those machines that are not assigned any spectrum in a certain scheduling time slot.

4.1. Scenario I: Without CCI. In this scenario, the performance of MSRA is compared with the SOTA algorithms including MSA [10], AASAA [9], and RCAA when CCI among CM2M devices is not considered. Therefore, we assume that $\mathrm{CM} 2 \mathrm{M}$ devices transmissions do not overlap with the transmission of other CM2M devices using the same channel.

For $M=30, \mathscr{L}$ increases by increasing the number of CM2M devices from 5 to 60 . Figure 4 shows that when the number of CM2M devices increases, the spectrum utilisation also increases in all three methods, but MSRA utilises all available whitespaces in various network loading conditions more efficiently than MSA, AASAA, and RCAA. This can be explained by the fact that, in case of higher $\mathscr{L}$, network can allocate better segments of spectrum to users because of higher multiuser diversity. In addition, because of using stochastic search method, MSRA achieves near to optimum solution in comparison to other SOTA solutions which are based on approximate algorithms. For MSRA, when $\mathscr{L}$ is higher than 3, CM2M network becomes saturated due to the lack of available spectrum. However, for the rest of the methods, there are still unassigned spectrum slices.

4.2. Scenario II: With CCI. In this scenario, CCI exists among CM2M devices and we compare our algorithm, MSRA, with AASAA and RCAA. As MSA inherently does not consider $\mathrm{CCI}$, for that reason, we do not include MSA for comparison.

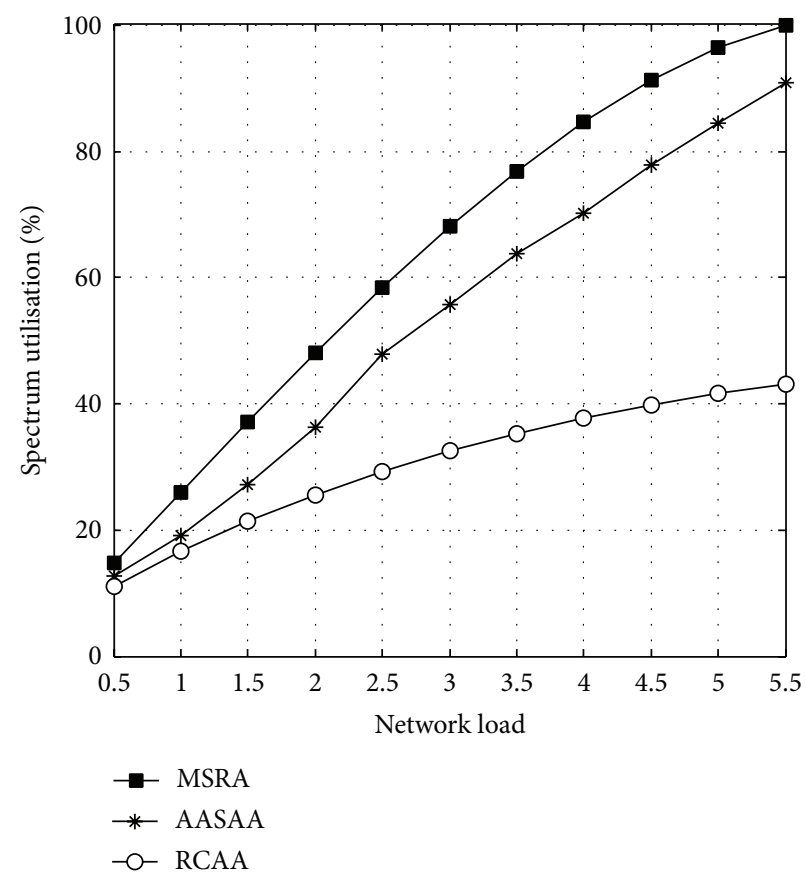

FIGURE 5: The impact of varying network load conditions on spectrum utilisation (scenario II: with CCI).

Figure 5 shows the spectrum utilisation, according to different network loads by increasing the number of CM2M devices from 5 to 55 when there are only seven available channels (i.e., $M=7$ ). As shown in Figure 5, MSRA outperforms AASAA and RCAA for different network loads. Similar to Scenario I, MSRA utilises TVWS even better than previous scenario, because some CM2M devices in network may reuse spectrum that is used by other devices in CM2M network.

Figure 6 represents the number of rejected CM2M devices when the network load increases. The number of rejected CM2M devices increases with the network load; MSRA has fewer numbers of rejected CM2M devices (or more satisfied devices) than AASAA and RCAA of different network loads. MSRA optimises spectrum utilisation by admitting devices with better channel quality to the network and allocates the spectrum resources effectively. Furthermore, MSRA does not assign any spectrum resources to the devices that has least contribution to overall network throughput. Figure 6 implies that MSRA increases the capacity of network (which is very vital for M2M networks because of a very large number of devices). Our approach may starve some of devices which are located far from the base station; in our future work, we will optimise network performance based on proportional fairness objective function to guarantee the fairness among devices.

4.3. Convergence of MSRA. Because of the nature of genetic programming, it is arguably impossible to make formal guarantees about the number of fitness evaluations needed for an algorithm to find an optimal solution. However, herein, computer experiments are performed to show the impact of 


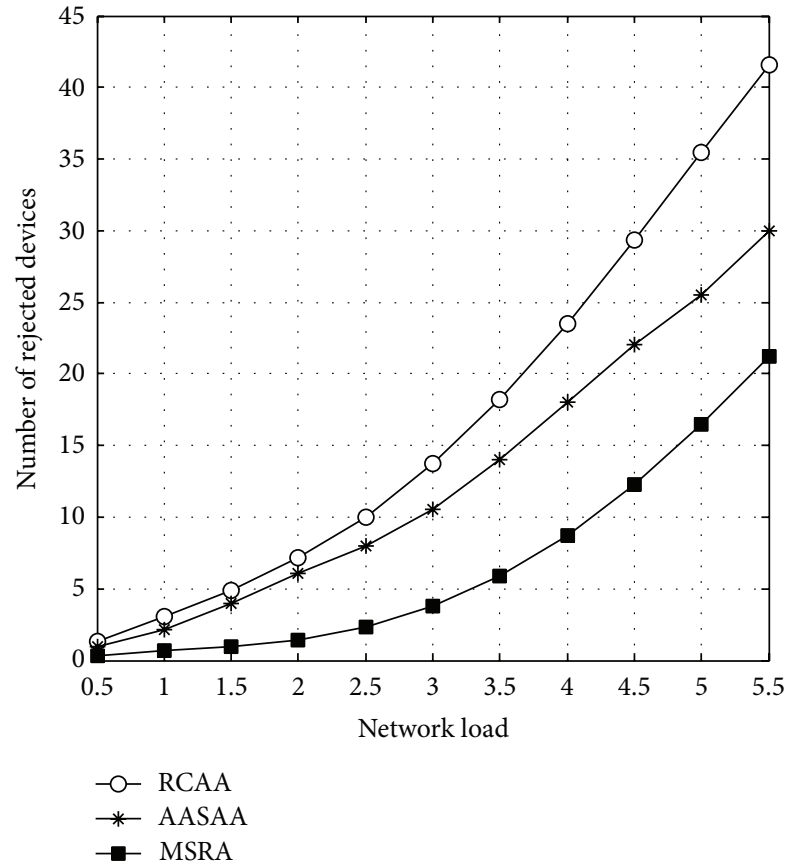

Figure 6: The impact of varying network load conditions on the number of rejected CM2M devices (scenario II: with CCI).

TABLE 2: System parameters.

\begin{tabular}{lc}
\hline Parameter & Value \\
\hline$M$ & 10 \\
$N$ & 200 \\
Processor & Intel Core i7-3667U 2.00 GHz \\
Memory (RAM) & 4 GB \\
OS & Windows 7 (64-bit) \\
Simulator & MATLAB R2011a (64-bit) \\
\hline
\end{tabular}

the number of generations on the performance of MSRA. The system parameters used in the section for simulation are listed in Table 2. For the purpose of convergence studies, we assume $N=200$ and $M=10$.

Figure 7 shows the best fitness value (MSRA) for a population in a different number of generations. As shown in Figure 7, the performance of algorithm is enhanced, when the number of generations increases; however this is at the cost of increased processing time. After roughly 34 generations, the fitness value saturates at optimal value which shows the effectiveness of using GA for spectrum assignment using spectrum aggregation.

Moreover, Figure 8 illustrates distribution of processing time for MSRA to find an optimal solution. As shown in Figure 8 , at $85 \%$ of time, MSRA finds an optimum solution in less than scheduling time slot (1 ms) and $15 \%$ takes more than scheduling time slot. Additionally, MSRA can be optimised to use fewer processor resources, so that it can execute more rapidly.

Furthermore, Lobo et al. [15] provided a theoretical and empirical analysis of the time complexity of traditional

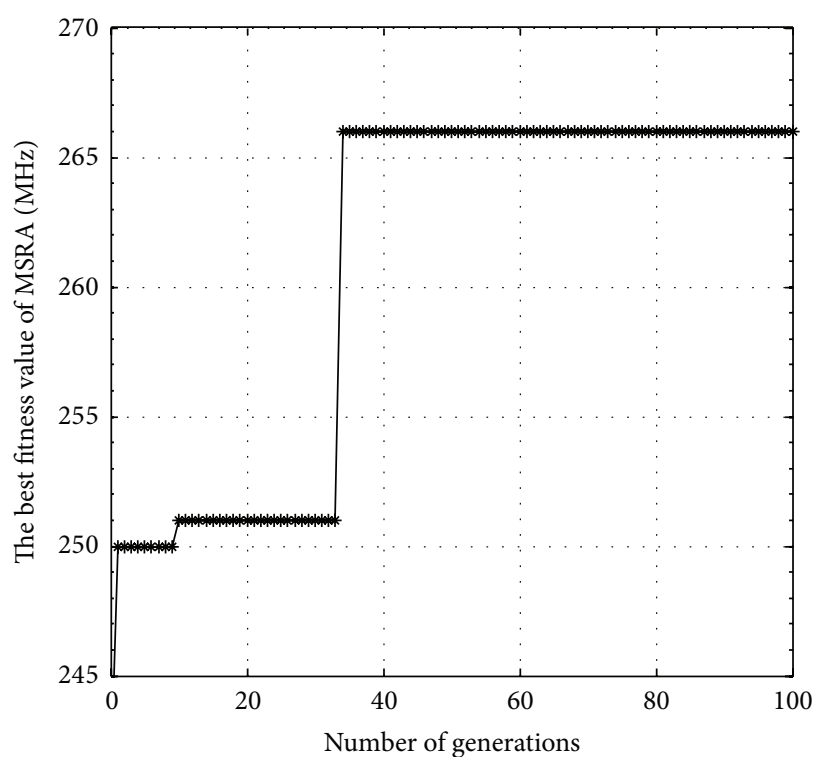

FIGURE 7: The impact of the number of generations on MSRA results.

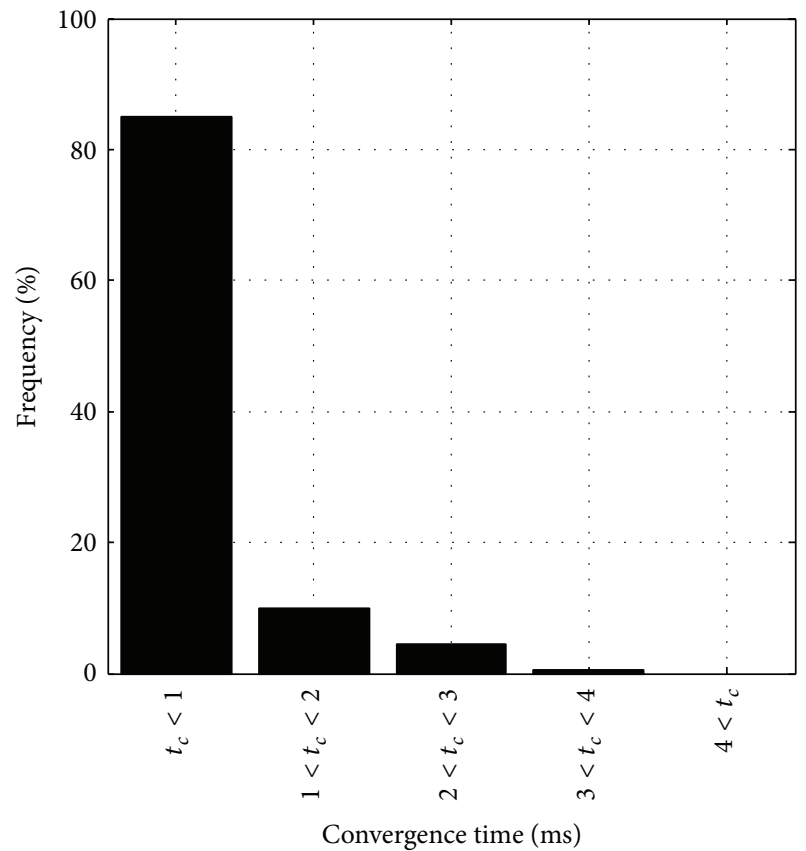

FIGURE 8: Distribution of processing time for MSRA to find an optimal solution.

simple GAs. According to [15], GA has time complexities of $\mathscr{O}\left(\sum_{i=1}^{N} \sum_{j=1}^{\mathscr{C}} l_{i, j}^{*}\right)$ which is dependent on length of each chromosome. The linear time complexity for GA occurs because the population sizing grows with the square root of chromosome length. The time complexity presented herein is for the worst-case scenario when the population size is assumed to be fixed and maximum of rest of generations. 


\section{Conclusion}

This article introduces an aggregation-aware spectrum assignment algorithm using genetic algorithm. The proposed algorithm maximises the spectrum utilisation to CM2M devices as a criterion to realise spectrum assignment. Moreover, the introduced algorithm takes into account the realistic constraints of co-channel interference and Maximum Aggregation Span. Performance of the proposed algorithm is validated by simulations and results are compared with algorithms available in the literature. The proposed algorithm decreases the number of rejected devices and improves the spectrum utilisation of CM2M network. Our algorithm increases the capacity of network which is very vital for M2M networks. For future work, we will investigate the impact of the various parameters used in genetic algorithm to solve the introduced utilisation function; in particular, population size, crossover rate, and mutation rate are the parameters that will be investigated in our study; in addition, we will further work on developing genetic algorithm based method to assign spectrum to CM2M devices in an energy-efficient manner.

\section{Competing Interests}

The authors declare that they have no competing interests.

\section{References}

[1] R. Lu, X. Li, X. Liang, X. Shen, and X. Lin, "GRS: the green, reliability, and security of emerging machine to machine communications," IEEE Communications Magazine, vol. 49, no. 4, pp. 28-35, 2011.

[2] "Cisco visual networking index: Global mobile data traffic forecast update 2014-2019 white paper," 2015, http://www.cisco .com/c/en/us/solutions/collateral/service-provider/visual-networking-index-vni/mobile-white-paper-cll-520862.html.

[3] S. Rostami, K. Arshad, and K. Moessner, "Order-statistic based spectrum sensing for cognitive radio," IEEE Communications Letters, vol. 16, no. 5, pp. 592-595, 2012.

[4] Y. Zhang, R. Yu, M. Nekovee, Y. Liu, S. Xie, and S. Gjessing, "Cognitive machine-to-machine communications: visions and potentials for the smart grid," IEEE Network, vol. 26, no. 3, pp. 6-13, 2012.

[5] M. Wylie-Green, "Dynamic spectrum sensing by multiband OFDM radio for interference mitigation," in Proceedings of the 1st IEEE International Symposium on New Frontiers in Dynamic Spectrum Access Networks (DySPAN '05), pp. 619-625, IEEE, Baltimore, Md, USA, November 2005.

[6] J. D. Poston and W. D. Horne, "Discontiguous OFDM considerations for dynamic spectrum access in idle TV channels," in Proceedings of the 1st IEEE International Symposium on New Frontiers in Dynamic Spectrum Access Networks (DySPAN '05), pp. 607-610, Baltimore, Md, USA, November 2005.

[7] R. Rajbanshi, A. M. Wyglinski, and G. J. Minden, "An efficient implementation of NC-OFDM transceivers for cognitive radios," in Proceedings of the 1st International Conference on Cognitive Radio Oriented Wireless Networks and Communications (CROWNCOM '06), pp. 1-5, Mykonos Island, Greece, June 2006.
[8] 3GPP, "LTE; evolved universal terrestrial radio access (e-utra); physical layer procedures," Tech. Rep. 3GPP TS 36.213 version 10.1.0 Release 10, 3GPP, 2010, http://www.3gpp.org.

[9] D. Chen, Q. Zhang, and W. Jia, "Aggregation aware spectrum assignment in cognitive ad-hoc networks," in Proceedings of the 3rd International Conference on Cognitive Radio Oriented Wireless Networks and Communications (Crown Com '08), pp. 16, May 2008.

[10] F. Huang, W. Wang, H. Luo, G. Yu, and Z. Zhang, "Predictionbased Spectrum aggregation with hardware limitation in cognitive radio networks," in Proceedings of the IEEE 71st Vehicular Technology Conference (VTC '10), pp. 1-5, May 2010.

[11] F. Ye, R. Yang, and Y. Li, "Genetic algorithm based spectrum assignment model in cognitive radio networks," in Proceedings of the 2nd International Conference on Information Engineering and Computer Science (ICIECS '10), pp. 1-4, Wuhan, China, December 2010.

[12] Z. Zhao, Z. Peng, S. Zheng, and J. Shang, "Cognitive radio spectrum allocation using evolutionary algorithms," IEEE Transactions on Wireless Communications, vol. 8, no. 9, pp. 4421-4425, 2009.

[13] K. Arshad, M. A. Imran, and K. Moessner, "Collaborative spectrum sensing optimisation algorithms for cognitive radio networks," International Journal of Digital Multimedia Broadcasting, vol. 2010, Article ID 424036, 20 pages, 2010.

[14] Y. Li, L. Zhao, C. Wang, A. Daneshmand, and Q. Hu, "Aggregation-based spectrum allocation algorithm in cognitive radio networks," in Proceedings of the IEEE Network Operations and Management Symposium (NOMS '12), pp. 506-509, IEEE, Maui, Hawaii, USA, April 2012.

[15] F. G. Lobo, D. E. Goldberg, and M. Pelikan, "Time complexity of genetic algorithms on exponentially scaled problems," in Proceedings of the Genetic and Evolutionary Computation Conference (GECCO '00), pp. 151-158, Morgan-Kaufmann, 2000. 

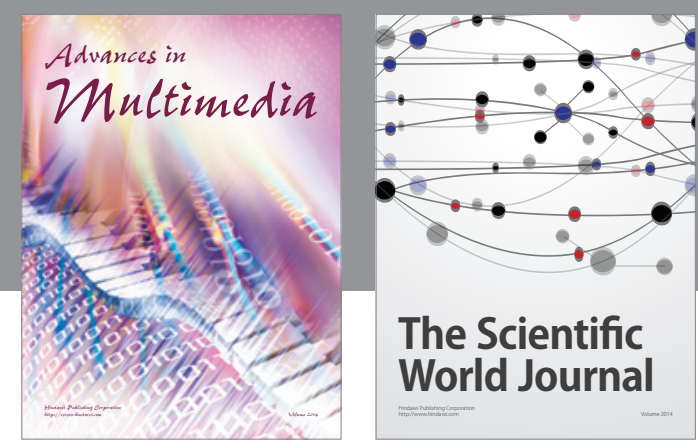

The Scientific World Journal
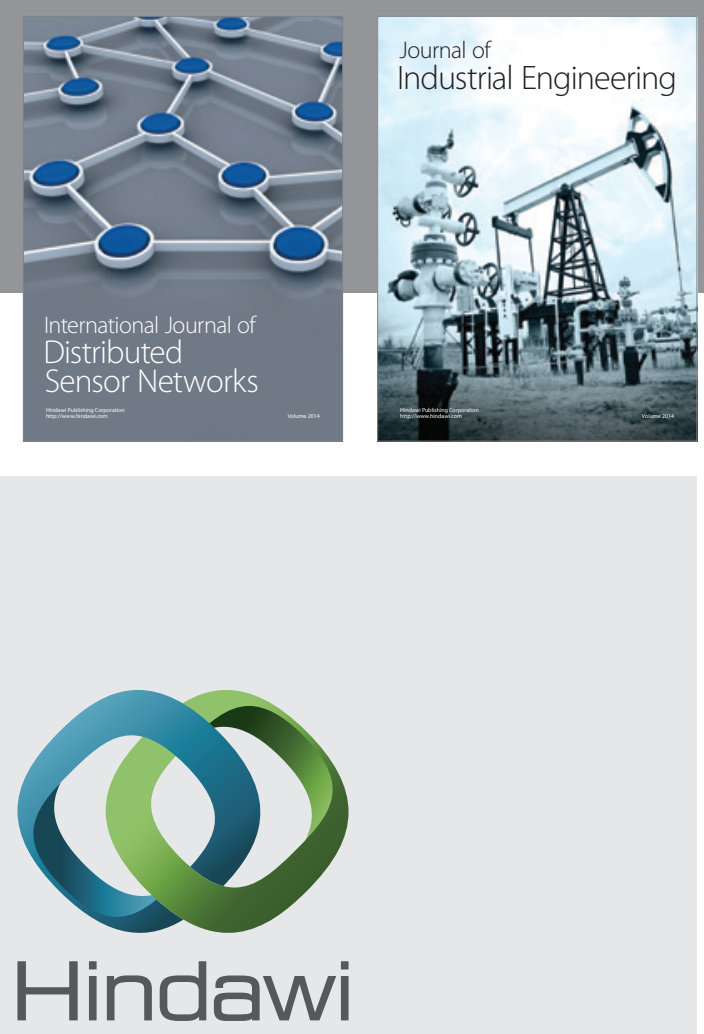

Submit your manuscripts at

http://www.hindawi.com

\section{Computer Networks} and Communications
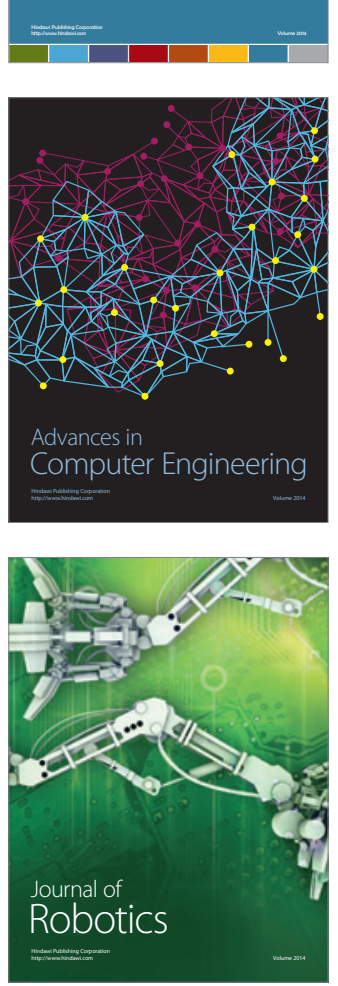
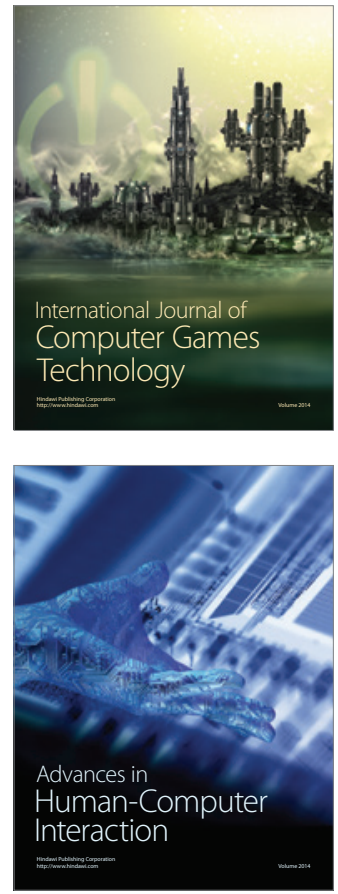
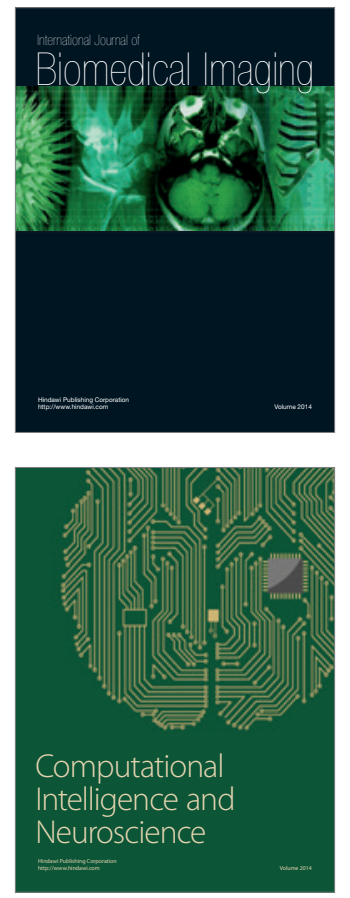
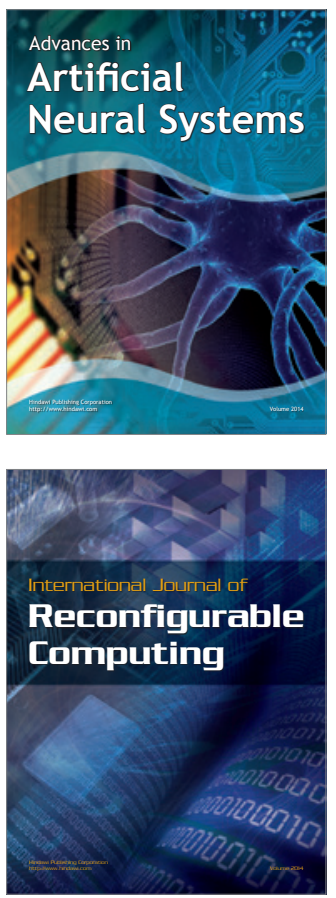
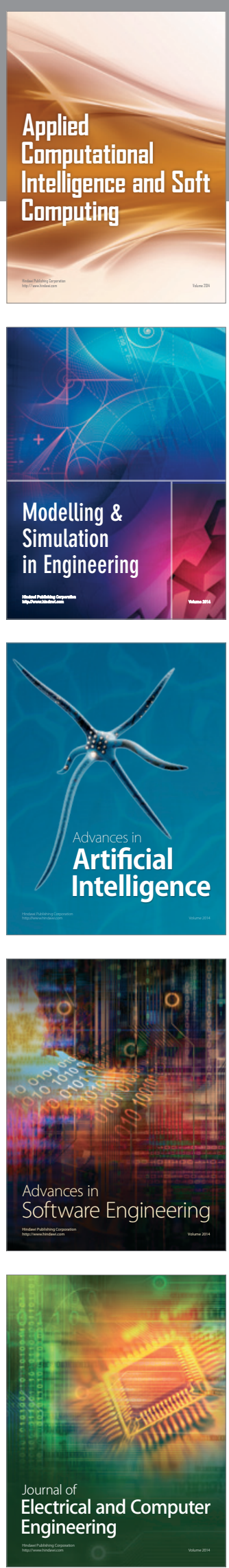